\title{
Impact of Cellular Genetic Make-up on Colorectal Cancer Cell Lines Response to Ellagic Acid: Implications of small interfering RNA
}

\author{
Amany I Yousef, Omar S El-Masry*, Mohamed A Abdel Mohsen
}

\begin{abstract}
Background: K-Ras activation is an early event in colorectal carcinogenesis and associated mutations have been reported in about $40 \%$ of colorectal cancer patients. These mutations have always been responsible for enhancing malignancy and silencing them is associated with attenuation of tumorigenicity. Among downstream effectors are the RAF/MEK/ERK and the PI3K/Akt signaling pathways. PI3K/Akt signaling leads to reduction of apoptosis, stimulated cell growth and enhanced proliferation. Ellagic acid (EA), a naturally occurring antioxidant, has recently emerged as a promising anti-cancer agent. Purpose: To evaluate the impact of cellular genetic makeup of two colon cancer cell lines with different genetic backgrounds, HCT-116 $\left(\mathrm{K} \cdot \mathrm{Ras}^{-} / \mathrm{ps3}^{+}\right)$and $\mathrm{Caco}^{-2}\left(\mathrm{~K}^{-} \cdot \mathrm{Ras}^{+} /\right.$ p53-), on response to potential anti-tumour effects of EA. In addition, the influence of K-Ras silencing in HCT116 cells was investigated. Materials and Methods: Cellular proliferation, morphology and cell cycle analysis were carried out in addition to Western blotting for detecting total Akt and p-Akt (at Thr308 and Ser473) in the presence and absence of different concentrations of EA. Cell proliferation was also assessed in cells transfected with different concentrations of K-Ras siRNA or incubated with ellagic acid following transfection. Results: The results of the present study revealed that EA exerts anti-proliferative and dose-dependent pro-apoptotic effects. Cytostatic and cytotoxic effects were also observed. p-Akt (at Thr308 and Ser473) was downregulated. Moreover, EA treatment was found to (i) reduce K-Ras protein expression; (ii) in cells transfected with siRNA and co-treated with EA, pronounced anti-proliferative effects as well as depletion of p-Akt (at Thr308) were detected. Conclusions: Cellular genetic makeup (K-Ras/p53-) was not likely to impose limitations on targeting EA in treatment of colon cancer. EA had a multi-disciplinary pro-apoptotic anti-proliferative approach, having inhibited Akt phosphorylation, induced cell cycle arrest and showed an anti-proliferative potential in HCT-116 cells (expressing mutant $K \cdot$ Ras).
\end{abstract}

Keywords: Ellagic acid - colorectal cancer - K-Ras - siRNA

Asian Pac J Cancer Prev, 17 (2), 743-748

\section{Introduction}

Colorectal cancer (CRC) is the third most prevalent malignancy worldwide (Umesalma and Sudhandiran, 2011). In Egypt, colorectal cancer is the 6th cancer both in males and females representing $4.5 \%$ and $3.6 \%$ of the total cancers (Zeeneldin, 2012).

$\mathrm{CRC}$ has been associated with a number of factors as diet, age, smoking, alcohol consumption and family history with heritable gene mutations (Yan et al., 2015). In this context, mutations in p53 with loss of heterozygosity coincide with adenoma to carcinoma transition in colorectal carcinogenesis (Sarasqueta et al., 2013). Additionally, K-Ras activation is an early event in colorectal carcinogenesis. Thus, mutations in $\mathrm{K}-\mathrm{R}$ as protein were reported in $40 \%$ of colorectal cancer patients. These mutations have always been responsible for enhancing malignancy and silencing them is associated with attenuation of tumorigenicity (Zenonos and Kyprianou, 2013). Among the downstream effectors signaling are the RAF/MEK/ERK and the PI3K/Akt pathways (Phipps et al., 2013; Temraz et al., 2015). PI3K/ Akt signaling leads to reduction of apoptosis, stimulated cell growth and enhanced proliferation (Danielsen et al., 2015). Akt Kinase (protein kinase B) is catalytically competent only if it is phosphorylated at Thr308 and Ser473 (Vadlakonda et al., 2013).

Besides targeting colon as well as other cancers with chemo- and radiotherapy, there is a pressing need for treatment that enhances cytotoxic effects with no or limited harm to normal cells. In this aspect, ellagic acid (EA), a naturally occurring antioxidant, has recently emerged as a promising anti-cancer agent (Vanella et al., 2013). Luca Vanella and colleagues (Vanella et al., 2013) 
have reported that EA had anti-proliferative and cytodifferentiating effects in prostate cancer cell types. A tight link between inflammation and colon cancer was reported (Klampfer, 2011). In this regard, the anti-inflammatory action of EA was reported by Khan and colleagues (Khan et al., 2013). Recently, it was reported that EA induced malignant cell death by compromising cellular metabolism and energy production (Mishra and Vinayak, 2014).

Accordingly, the present research work was planned to evaluate the impact of cellular genetic make-up of HCT-116 (K-Ras $\left./ \mathrm{P}^{-} 3^{+}\right)$and Caco-2 (K-Ras $\left.{ }^{+} / \mathrm{P}^{-} 3^{-}\right)$on response to the potential anti-tumour effect of EA. Also, the potential influence of K-Ras silencing in HCT-116 cells was investigated.

\section{Materials and Methods}

Materials: Ellagic acid was purchased from SigmaAldrich (Poole, Dorset, UK). Dulbecco's minimal essential medium (DMEM) was obtained from Gibco BRL (Paisley, UK) and foetal calf serum (FCS) from Autogenbioclean (UK Ltd.). Phosphate buffered saline (PBS) and trypsin/EDTA were obtained from Sigma Aldrich (Irvine, Ayrshire, UK), Cell titer 96® Aqueous One Solution cell proliferation assay was from Promega (Madison, WI, USA). T25 and T75 tissue culture flasks were from Nunc ${ }^{\mathrm{TM}}$ (Denmark) and tissue culture sterile 6-, 24- and 96-well plates and Petri dishes were from Costar ${ }^{\circledR}$, USA. Phospho-Akt (p-Ser374 and p-Thr308), total Akt, K-Ras and actin antibodies along with K-Ras siRNA (h) (SC-35731) were all purchased from Santa Cruz biotechnology (Dallas, Texas, USA). Western blotting alkaline phosphatase calorimetric detection kit was purchased from BioRad (CA, USA and München, Germany). TBST and the transfer buffer were prepared from their components.

Colon cancer cell lines with different genetic backgrounds regarding p53 and Ras status (Caco-2 (p53 mutant and $\mathrm{K}^{-} \mathrm{Ras}^{\mathrm{wt}}$ ) and HCT-116 (p53wt and K-Ras mutant)) were kindly provided by Medical Technology Center, Medical Research institute, University of Alexandria, Egypt.

Cell culture: Colon cancer cells were grown in a continuous monolayer culture in T75 top filtered sterile tissue culture flasks inside a sterile humidified incubator at $37^{\circ} \mathrm{C}$ with $5 \% \mathrm{CO}_{2}$ in air. Cells were sub-cultured as required for maintenance and plating.

Western blotting: Cell lines were sub-cultured, counted using a hemocytometer and plated at $1 \times 10^{6}$ cells in appropriate tissue culture plates and incubated at $37^{\circ} \mathrm{C}$ with $5 \% \mathrm{CO}_{2}$ in a humidified atmosphere. Cells were incubated with ellagic acid $(30 \mu \mathrm{g} / \mathrm{ml})$ or $(100 \mu \mathrm{g} /$ $\mathrm{ml}$ ) (or as appropriately described in the Figure legends) with corresponding controls for 1 hour. Cell lysates were collected from the control and treated cells, followed by a protein assay. Equal protein concentrations from lysates of control and treated cells were loaded at a maximum volume of $30 \mu \mathrm{l} /$ well into wells of freshly prepared polyacrylamide SDS-gels. Proteins were then separated by electrophoresis and transferred to a nitrocellulose membrane. The membrane was then blocked overnight in $10 \%$ blocking solution (BSA in TBST) prior to being incubated overnight at $4^{\circ} \mathrm{C}$ with phospho-Akt antibodies (targeting p-Ser473 and p-Thr308 amino acid residues), total Akt or actin antibodies. The membrane was washed in TBST four times for $15 \mathrm{~min}$ each and incubated with polyclonal anti-rabbit IgG antibody provided with alkaline phosphatase colorimetric detection kit. The membrane was washed again four times prior to detection. Silencing of $\mathrm{K}$-Ras was also confirmed using Western blotting.

Cell proliferation: Cells were sub-cultured, counted, plated at a density of $7 \times 10^{3}$ cells /well in sterile 96 well plates and incubated overnight at $37^{\circ} \mathrm{C}$ with $5 \% \mathrm{CO}_{2}$ in air. The following day, serial concentrations of ellagic acid $(0-200 \mu \mathrm{g} / \mathrm{ml})$ were added or transfection protocol was commenced for K-Ras silencing. The number of viable cells was estimated following 72 hours of incubation via their metabolic activity using the Aqueous One assay kit (Promega, Maddison, USA), which assesses the ability of cells to reduce the colorless tetrazolium, producing redcolored formazan, whose color was measured at 490 and $630 \mathrm{~nm}$ using a Biotek ELX800 Microplate reader (Biotek Instruments, Winooski, VT, USA). Cell proliferation was similarly assessed in cells transfected with different concentrations of K-Ras siRNA or those incubated with ellagic acid following transfection.

Microscopy: The cell lines were treated with ellagic acid (30 or $100 \mu \mathrm{g} / \mathrm{ml})$ after being sub-cultured, counted and plated at $2 \times 10^{5}$ cells /well in sterile tissue culture 6 well plates. Treated cells and corresponding controls (incubated with DMEM) were examined for morphological features of apoptosis following an incubation period of 48 hours under the Xli-Cap software linked light microscope (Xli, CETi, UK).

Cell cycle analysis: $1 \times 10^{6}$ cells of sub-cultured cells were seeded in $100 \mathrm{~mm}$ sterile tissue culture Petri dishes (Thermo scientific, UK) and incubated overnight at $37^{\circ} \mathrm{C}$ and $5 \% \mathrm{CO}_{2}$ to adhere. Zero time cells were washed with PBS and combined with non-adherent cells. Cells were washed in ice cold PBS for 10 minutes at $200 \mathrm{~g}$ and $4^{\circ} \mathrm{C}$. Cells were permeabilized in $1 \mathrm{ml} 70 \%$ ethanol overnight at $4^{\circ} \mathrm{C}$. Permeabilized cells were washed twice as mentioned above and $300 \mu 1$ of DNA staining fluorochrome, propidium iodide (PI (BD pharminogen ${ }^{\mathrm{TM}}$, \#556463) was added. Labelled cells were incubated overnight at $4^{\circ} \mathrm{C}$. The emission spectra of PI (read at FL3H) were measured using flow cytometry. Cells treated with ellagic acid (30 or 100 $\mu \mathrm{g} / \mathrm{ml}$ ) were incubated with their corresponding controls at $37^{\circ} \mathrm{C}$ and $5 \% \mathrm{CO}_{2}$ for 48 hours and were similarly stained with PI. Forward and side scatter (FSC and SCC) plots were created and the output was a histogram of PI emission spectra (FL3H) classifying cells into 4 populations based on their DNA content (subG1 (apoptotic or dying cells), G1, S and M).

K-Ras silencing: K-Ras silencing in HCT-116 colon cancer cells was carried out according to the protocol provided with K-Ras siRNA system (SC-SC-35731, Santa Cruz biotechnology). K-Ras gene silencing was confirmed by Western blotting using K-Ras antibody (C17; SC-522, Santa Cruz biotechnology). 


\section{Results}

Ellagic acid exerted anti-proliferative effect on Caco-2 and HCT-116 cells

Increasing doses of ellagic acid resulted in a dosedependent anti-proliferative effect in $\mathrm{CaCo}-2$ and HCT116 colon cancer cells. This anti-proliferative action was significant when compared to control cells $(100 \%$ proliferation). $\mathrm{CaCo}-2$ cells appeared to be more sensitive to the action of ellagic acid at high concentrations; though the difference between cells was not significant (Figure 1).

\section{Microscopy}

Incubation of cells with ellagic acid induced typical morphological changes of apoptosis including membrane blebbing and eventually fragmentation of cells. High concentration of ellagic acid was associated with more apoptotic cells. Of note, there was no differential response to ellagic acid despite the different genetic makeup of cells (Figure 2).

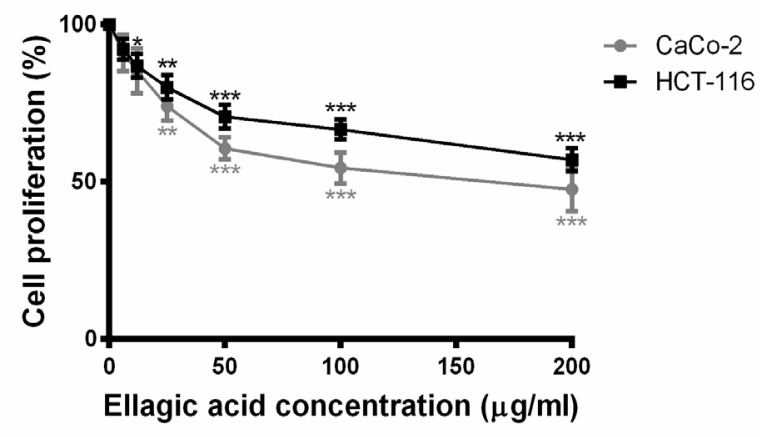

Figure 1. Effect of Ellagic Acid on Caco-2 and HCT-116 Cell Proliferation. Increasing Concentrations of Ellagic Acid induced a Significant Decline in Cell Proliferation of CaCo-2 and HCT-116 Cell Types when Compared to the Vehicle Control $(100 \%){ }^{*}=\mathrm{p} \leq 0.05, * *=\mathrm{p} \leq 0.01$ and $* * *=\mathrm{p} \leq 0.001(\mathrm{n}=6)$
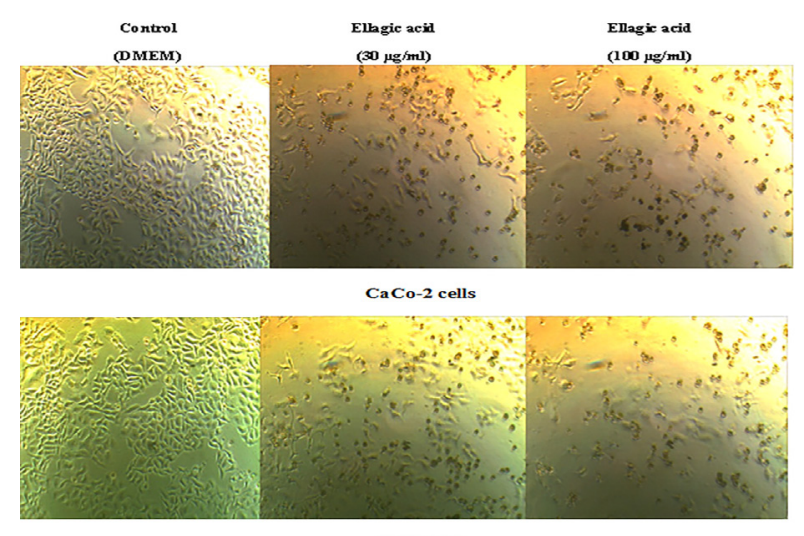

HCT-116

Figure 2. Photomicrographs Confirmed the Antiproliferative Influence of Ellagic acid on Both Cell types. Apparent Changes in Cell Morphology were Observed in CaCo-2 and HCT-116 Cells Following Incubation with Ellagic Acid (30 or $100 \mu \mathrm{g} / \mathrm{ml}$ ) for 48 hours. A remarkable loss of proper cell architecture was observed especially at the highest concentration of the drug
Ellagic acid treatment induced cell cycle arrest

flow cytometry analysis showed that ellagic acid elicited both cytotoxic and cytostatic effects through increasing the number of cells in the Sub-G1 phase in CaCo- 2 and HCT-116 cells, though the effect was more significant in HCT-116 cells (Figure 3A) with augmented PI staining. In addition, cell treatment halted cell cycle progression at $\mathrm{G} 1$ and $\mathrm{S}$ phases in both cell types, though the effect was significant in $\mathrm{CaCo}-2$ cells only. There was a significant increase in the number of cells recorded in $\mathrm{S}$ phase in treated CaCo- 2 cells $(100 \mu \mathrm{g} / \mathrm{ml})$ when compared
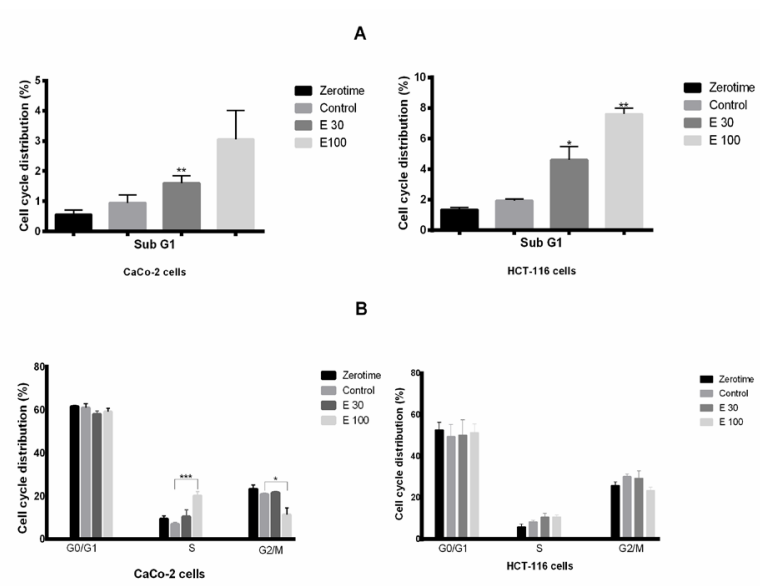

Figure 3. Effect of Ellagic Acid on Cell Cycle Progression. CaCo-2 and HCT-116 were Incubated with Ellagic Acid (E) (30 or $100 \mu \mathrm{g} / \mathrm{ml}$ ) or DMEM for 48 hours. Cell cycle analysis showed that ellagic acid, at its highest dose; resulted in an apoptotic effect (A) as well as arresting cells in G1 phase. Ellagic acid increased the number of cells in S phase (B), whilst a concomitant decrease in the number of cells in $\mathrm{G} 2 / \mathrm{M}$ phase was observed (significant in $\mathrm{CaCo}-2$ cells only). The apoptotic effect appears to be more significant In HCT-116 cells. $*=p \leq 0.05, * *=p \leq 0.01$ and $* * *=p \leq 0.001(n=3)$

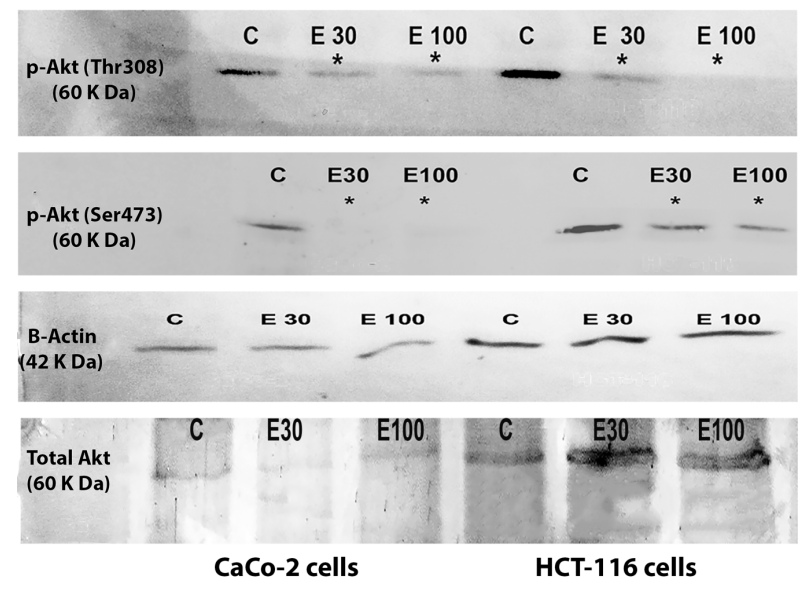

Figure 4. Effect of Ellagic Acid on Akt Phosphorylation. Treatment of CaCo-2 and HCT-116 Cells with Ellagic Acid (30 or $100 \mu \mathrm{g} / \mathrm{ml}$ ) Inhibited Akt Phosphorylation (at Thr308 and Ser473) Significantly in Both Cell Lines. There was no significant difference in the level of actin or total Akt between control and treated cells suggesting that the inhibitory effect was not a result of difference in protein levels. $*=\mathrm{p} \leq 0.05 . \mathrm{C}=$ control, $\mathrm{E} 100=$ ellagic acid $(100 \mu \mathrm{g} / \mathrm{ml})$ and $\mathrm{E} 30=$ ellagic acid $(30 \mu \mathrm{g} / \mathrm{ml})$. Blots are representative of 3 independent experiments 
to the corresponding DMEM-treated control cells. A concomitant significant reduction in the number of G2/M cell population was also reported when compared to the corresponding control (Figure 3B). Although a similar increase in the number of $S$ phase cells along with a concomitant reduction in $\mathrm{G} 2 / \mathrm{M}$ cells was recorded in HCT-116 cells, the difference was not significant when compared to the corresponding control cells.

\section{Ellagic acid suppressed Akt phosphorylation at Thr308} and $\operatorname{Ser} 473$

Incubation of CaCo-2 and HCT-116 cells with ellagic acid (30 or $100 \mu \mathrm{g} / \mathrm{ml}$ ) for one hour depleted Akt phosphorylation significantly. There was no difference neither in actin nor total Akt levels between ellagic acidtreated cells and their corresponding controls (Figure 4).
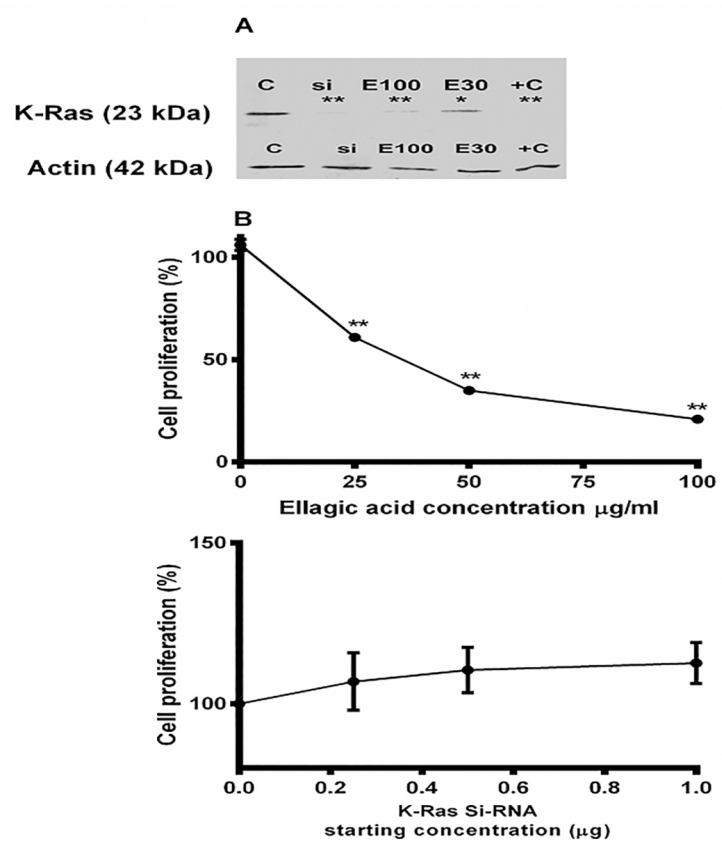

C

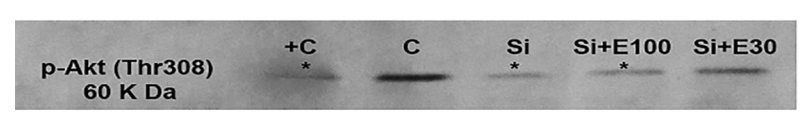

Figure 5. Silencing of KRas and Treatment of Transfected HCT-116 Cells with Ellagic Acid Inhibited Cell Proliferation and Akt Phosphorylation at Thr308. HCT-116 Cells were Transfected with $1 \mu \mathrm{g} \mathrm{K}$-Ras siRNA and Western Blotting Confirmed Successful Silencing in Transfected Cells. Separate sets of cells were incubated with Ellagic acid for 24 hours and this resulted in a significant reduction of K-Ras levels when compared to the control cells (A). K-Ras silencing (0.25 - $1 \mu \mathrm{g}$ siRNA) had no significant impact on cell proliferation. Incubation of transfected cells ( $1 \mu \mathrm{g}$ siRNA) with ellagic acid inhibited cell proliferation significantly when compared to the corresponding transfected control cells (B). K-Ras silencing alone or combined with ellagic acid administration $(100 \mu \mathrm{g} / \mathrm{ml})$ suppressed Akt phosphorylation significantly upon comparison with the corresponding controls (C) $. \mathrm{C}=$ control, $\mathrm{si}=\mathrm{K} \cdot \mathrm{R}$ as siRNA, E100 and E30 = ellagic acid $(100 \mu \mathrm{g} / \mathrm{ml}$ and $30 \mu \mathrm{g} / \mathrm{ml})$ and $+\mathrm{C}=$ positive control. $*=\mathrm{p} \leq 0.05$ and $* *=\mathrm{p} \leq 0.01(\mathrm{n}=3)$
$K^{-R a s}$ silencing had no effect on HCT-116 cell proliferation

A successful K-Ras silencing was confirmed by Western blotting blots. A remarkable observation was the ability of ellagic acid to reduce the levels of K-Ras significantly when compared to the corresponding controls (Figure 5 A). Transfection of HCT-116 cells with siRNA of K-Ras (starting concentrations $0.25-1 \mu \mathrm{g}$ ) showed no effect on cell proliferation when compared to the nontransfected cells (100\% cell proliferation). Incubation of K-Ras siRNA-transfected cells with increasing doses of ellagic acid resulted in an anti-proliferative influence, which was similar to that reported in Figure 1 (Figure 5 B). In addition, K-Ras silencing and co-treatment with ellagic acid resulted in a significant inhibition of Akt phosphorylation at Thr308 (Figure 5 C).

\section{Discussion}

The main purpose of chemotherapy is to target proliferating cells to induce cellular death pathways including apoptosis. In this aspect, EA treatment showed an anti-proliferative effect on both cell types independently on the p53 or Ras status (Figure 1). Previously, antiproliferative effect of EA on different types of cancer cells has been reported. Kao and colleagues (Kao et al., 2012) have reported a similar anti-proliferative influence of EA on four different colorectal cancer cell types. Also, differential anti-proliferative influence was reported for EA on breast cancer cells, MCF-7 and MDA-MB-231 (Hyun-Ah Kim, 2009). The anti-proliferative effect of EA on CaCo-2 and HCT-116 cells was associated with apparent changes of cell morphology identical to that seen in apoptosis (Figure 2). Zhang and coworkers (Zhang et al., 2014) have demonstrated apoptosis as a mode of action of EA in several cancer cell lines. Moreover, EA has also modulated cell cycle progression in both cell lines (Figure 3). Its treatment resulted in an increase in number of apoptotic cells significantly and induced cell cycle arrest at G1 phase. This was also accompanied by increasing number of cells in $\mathrm{S}$ phase and decreasing number of mitotic cells. Similar observations have been reported by Ramirez study (Ramirez de Molina et al., 2015), which reported a similar effect of EA derivative, 4,4'-di-O-methyl ellagic acid (4,4'-DiOMEA) on colon cancer cells without derailing the growth of normal colon cells. Juan Carlos and colleagues (Espin et al., 2013) have stated that a mixture of EA and its metabolites inhibited CaCo-2 cancer cell proliferation through cell cycle arrest associated with modulation of cell cycle regulatory genes. Accordingly, the results of the present work are in agreement with the previously mentioned studies. All of these results may point to the anti-proliferative and proapoptotic role of EA.

Activated PI3K/Akt pathway plays a central role in colon tumorigenesis (Aggarwal et al., 2004; Chappell et al., 2011). It has been reported that EA inhibited chemically-induced colon carcinogenesis by a mechanism involved inhibition of Akt phosphorylation at Ser473 (Umesalma and Sudhandiran, 2011). Similar inhibitory effect of EA on Akt phosphorylation at Thr308 and Ser473 has been observed (Figure 4). This, at least in part, may 
have its contribution in inhibiting cell proliferation by EA. In this regard, recent data provided evidence on the role of Akt inactivation (inhibition of phosphorylation) in mediating EA anti-proliferative influence in HCT-15 colon cancer cells (Umesalma et al., 2015). The capability of p-Akt to phosphorylate/activate m-TOR has been described in many cancers cells (Pratheeshkumar et al., 2012; Diersch et al., 2013; Vinayak and Carlson, 2013). Inhibiting $\mathrm{m}$-TOR activation is a potential target for developing anti-cancer therapeutics (Pratheeshkumar et al., 2012; Fagone et al., 2013). Therefore, it could be argued that the anti-proliferative effect of EA, through reducing the Akt phosphorylation, subsequently will reduce the downstream signaling of the PI3K/Akt pathway. This could be supported the observation that EA can potentiate sensitivity of colorectal cancer cells to 5-fluorouracil (5-FU) (Kao et al., 2012).

In an attempt to further investigate the efficacy of EA in HCT-116, the effect of mutant K-Ras silencing on cell proliferation in the presence or absence of EA has been investigated. Results showed that siRNA against K-Ras had no effect on cell proliferation. However, a significant inhibition of cell proliferation has been observed on transfection of cells with K-Ras siRNA and treatment with EA (Figure 5 A and B). Teimoori-Toolabi and colleagues (Teimoori-Toolabi et al., 2015), recently reported that mutant as well as wild-type $\mathrm{K}$-Ras would sensitize cells to 5-FU treatment, whilst silencing mutant and wild-type $\mathrm{K}-\mathrm{Ras}$ in HCT-116 increased resistance to 5-FU. This might explain our finding that silencing mutant $\mathrm{K}$-Ras in HCT-116 did not have an influence on cell proliferation (Figure 5B). This may point out to the impact of EA on other biochemical pathways to overcome consequences of cellular resistance following silencing of mutant K-Ras. Treating cells with EA not only decreased cellular proliferation, but also caused further downregulation in the expression of K-Ras protein itself (Figure $5 \mathrm{~A}$ ). In this aspect, Tahir and colleagues (Tahir et al., 2015) demonstrated that combining ginger and Gelam honey both are characterized with high content of polyphenols as EA downregulated K-Ras gene expression in HCT-29 cell line. K-Ras silencing was accompanied by downregulation of Akt phosphorylation (at Thr308) when compared to control cells, confirming the role of activated K-Ras in activating the PI3K/Akt pathway (Knickelbein and Zhang, 2015). Moreover, it could be suggested that treatment with EA with silencing mutant K-Ras, completely depleted Akt phosphorylation at Thr308 (Figure 5 C).

In conclusion, cellular genetic makeup (Ras-/p53-) was not likely to impose limitations on targeting EA in treatment of colon cancer. EA had a multi-disciplinary proapoptotic anti-proliferative approach, having inhibited Akt phosphorylation, induced cell cycle arrest and showed an anti-proliferative potential in HCT-116 cells (expressing mutant K-Ras).

\section{References}

Aggarwal BB, Takada Y, Oommen OV (2004). From chemoprevention to chemotherapy: common targets and common goals. Expert Opin Investig Drugs, 13, 1327-38.
Ahmed A Zeeneldin MMS, Ibrahim A Seif El-din , Sara A Frag (2012). Colorectal carcinoma in gharbiah district, Egypt:

Comparison between the elderly and non-elderly. J Solid Tumors, 2, 13-23.

Chappell WH, Steelman LS, Long JM, et al (2011). Ras/Raf/ MEK/ERK and PI3K/PTEN/Akt/mTOR inhibitors: rationale and importance to inhibiting these pathways in human health. Oncotarget, 2, 135-64.

Danielsen SA, Eide PW, Nesbakken A, et al (2015). Portrait of the PI3K/AKT pathway in colorectal cancer. Biochim Biophys Acta, 1855, 104-21.

Diersch S, Wenzel P, Szameitat M, et al (2013). Efemp1 and p27(Kip1) modulate responsiveness of pancreatic cancer cells towards a dual PI3K/mTOR inhibitor in preclinical models. Oncotarget, 4, 277-88.

Espin JC, Larrosa M, Garcia-Conesa MT, et al (2013). Biological significance of urolithins, the gut microbial ellagic Acidderived metabolites: the evidence so far. Evid Based Complement Alternat Med, 2013, 270418.

Fagone P, Donia M, Mangano K, et al (2013). Comparative study of rapamycin and temsirolimus demonstrates superimposable anti-tumour potency on prostate cancer cells. Basic Clin Pharmacol Toxicol, 112, 63-9.

Hyun-Ah Kim R-AL, Byung In Moon, Kuk-Jin Choe (2009). Ellagic acid shows different anti-proliferative effects between the MDA-MB-231 and MCF7 human breast cancer cell lines. J Breast Cancer, 12, 85-91.

Kao TY, Chung YC, Hou YC, et al (2012). Effects of ellagic acid on chemosensitivity to 5 -fluorouracil in colorectal carcinoma cells. Anticancer Res, 32, 4413-8.

Khan MK, Ansari IA, Khan MS (2013). Dietary phytochemicals as potent chemotherapeutic agents against breast cancer: Inhibition of NF-kappaB pathway via molecular interactions in rel homology domain of its precursor protein p105. Pharmacogn Mag, 9, 51-7.

Knickelbein K, Zhang L (2015). Mutant KRAS as a critical determinant of the therapeutic response of colorectal cancer. Genes Dis, 2, 4-12.

Phipps AI, Buchanan DD, Makar KW, et al (2013). KRASmutation status in relation to colorectal cancer survival: the joint impact of correlated tumour markers. Br J Cancer, 108, 1757-64.

Pratheeshkumar P, Budhraja A, Son YO, et al (2012). Quercetin inhibits angiogenesis mediated human prostate tumor growth by targeting VEGFR- 2 regulated AKT/mTOR/P70S6K signaling pathways. PLoS One , 7, 47516.

Ramirez de Molina A, Vargas T, Molina S, et al (2015). The ellagic acid derivative 4,4'-di-O-methylellagic acid efficiently inhibits colon cancer cell growth through a mechanism involving WNT16. J Pharmacol Exp Ther, 353, 433-44.

Sarasqueta AF, Forte G, Corver WE, et al (2013). Integral analysis of $\mathrm{p} 53$ and its value as prognostic factor in sporadic colon cancer. BMC Cancer, 13, 277.

Tahir AA, Sani NF, Murad NA, et al (2015). Combined ginger extract \& Gelam honey modulate Ras/ERK and PI3K/AKT pathway genes in colon cancer HT29 cells. Nutr J, 14, 31 .

Teimoori-Toolabi L, Hashemi S, Azadmanesh K, et al (2015). Silencing the wild-type and mutant K-ras increases the resistance to 5-flurouracil in HCT-116 as a colorectal cancer cell line. Anticancer Drugs, 26, 187-96.

Temraz S, Mukherji D, Shamseddine A (2015). Dual Inhibition of MEK and PI3K Pathway in KRAS and BRAF Mutated Colorectal Cancers. Int J Mol Sci, 16, 22976-88.

Umesalma S, Nagendraprabhu P, Sudhandiran G (2015). Ellagic acid inhibits proliferation and induced apoptosis via the Akt signaling pathway in HCT-15 colon adenocarcinoma cells. 
Amany I Yousef et al

Mol Cell Biochem, 399, 303-13.

Umesalma S, Sudhandiran G (2011). Ellagic acid prevents rat colon carcinogenesis induced by 1,2 dimethyl hydrazine through inhibition of AKT-phosphoinositide-3 kinase pathway. Eur J Pharmacol, 660, 249-58.

Vadlakonda L, Dash A, Pasupuleti M, et al (2013). The Paradox of Akt-mTOR Interactions. Front Oncol, 3, 165.

Vanella L, Di Giacomo C, Acquaviva R, et al (2013). Effects of ellagic Acid on angiogenic factors in prostate cancer cells. Cancers (Basel), 5, 726-38.

Vinayak S, Carlson RW (2013). mTOR inhibitors in the treatment of breast cancer. Oncology, 27, 38-44.

Yan WF, Wu G, Sun PC, et al (2015). P53 mutations occur more commonly than KRAS mutations in colorectal adenoma. Int $J$ Clin Exp Med, 8, 1370-5.

Zenonos K, Kyprianou K (2013). RAS signaling pathways, mutations and their role in colorectal cancer. World $J$ Gastrointest Oncol, 5, 97-101.

Zhang HM, Zhao L, Li H, et al (2014). Research progress on the anticarcinogenic actions and mechanisms of ellagic acid. Cancer Biol Med, 11, 92-100. 